\title{
A Method for the Measurement of Temperature Based on Neural Network PID
}

\section{Gaoli Chen}

Zhengzhou University of Industrial Technology, Zhengzhou, He’nan 451100, China

amy000@163.com

Keywords: BP neural network, temperature control, PID control

Abstract: Environment for plant growth is difficult to establish precise mathematical model. The conventional control methods are difficult to be well controlled, and put forward a neural network PID control temperature on the growth environment of plant. In this paper, taking the lettuce as an example, using MATLAB to simulate the PID control and PID control of BP neural network, the results proved that PID control of BP neural network has small overshoot, fast response speed and good stability compared with the traditional PID control, and better controlled temperature changing with the target temperature.

\section{Introduction}

Because neural network can adapt to the non-linearity, uncertainty and complexity of the control system, showing good robustness and adaptability, it is more and more popular with people in the temperature control ${ }^{[1]}$. When the mathematical model of the controlled object is changing, non-linear time, PID parameters is not easy to make adjustments according to their actual situation, affecting the quality of control, making the control quality of control system decreased, especially in industrial processes with time-delay, conventional PID control is more difficult to meet the requirements of control precision. The neural network has self-organization, self-learning, self-adaption capacity. PID controller of BP neural network is proposed, the artificial neural network and traditional PID control being combined, working together to improve the control quality. PID control for neural network is an improved control method of neural network is applied to the PID control and the controller with the traditional PID which combines. It is an improvement and optimization of the traditional PID control.

\section{Composition of control system for plant growth cabinet}

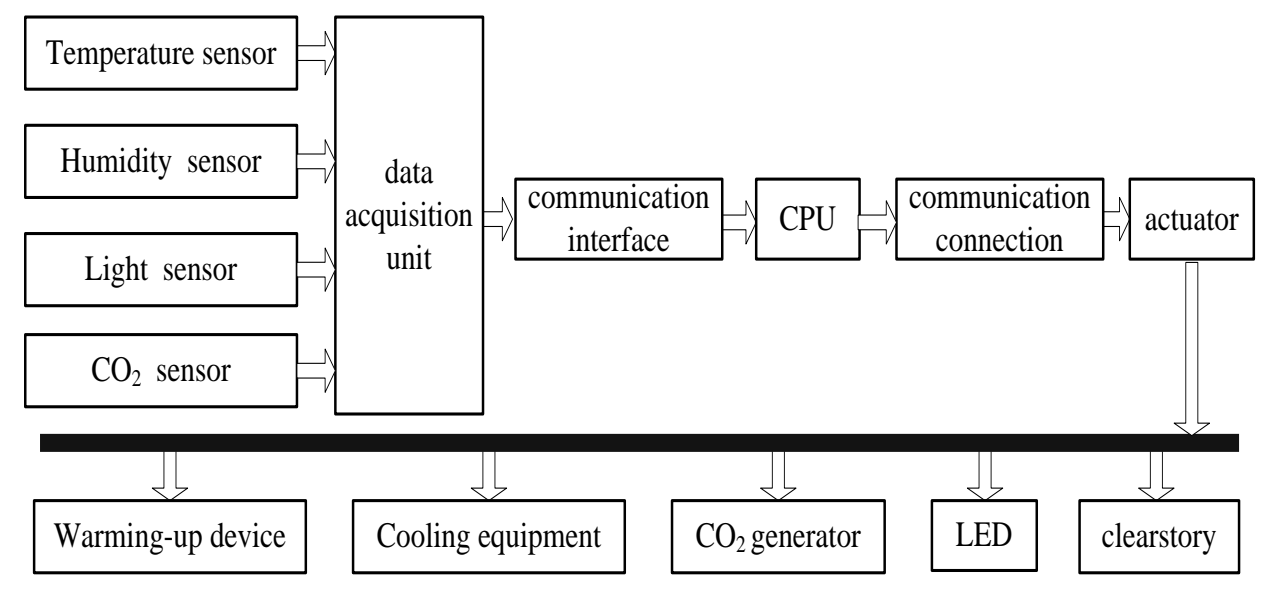

Fig. 1 Schematic diagram of the system composition

The multi-source sensor is arranged in the same growth cabinet at different location and height ${ }^{[2]}$, while the information of each growth environment factor in cabinet through the sensor is transferred to the computer, and the computer will analyze these signals that converted from the sensor, and can show the environmental situation of greenhouse, so expect to make judgment, and make the 
environment of crop growth make a change. Schematic diagram of the system composition is shown in Fig. 1.

The structure of plant growth cabinet was shown in Fig. 2. Top of the cabinet is LED lights that controlled by an artificial matching red and blue, and the ratio of red and blue can being adjusted to simulate the natural growth environment of plants. Cabinet also has the regulating the temperature of the heater, fan, regulating $\mathrm{CO}_{2}$ concentration of $\mathrm{CO}_{2}$ generator and adjusting the humidity of ventilation fans, humidifiers. Cabinet distribute four temperature sensors in the upper half part and the lower half part, a total of eight sensors, measuring and recording the temperature in the growth cabinet.

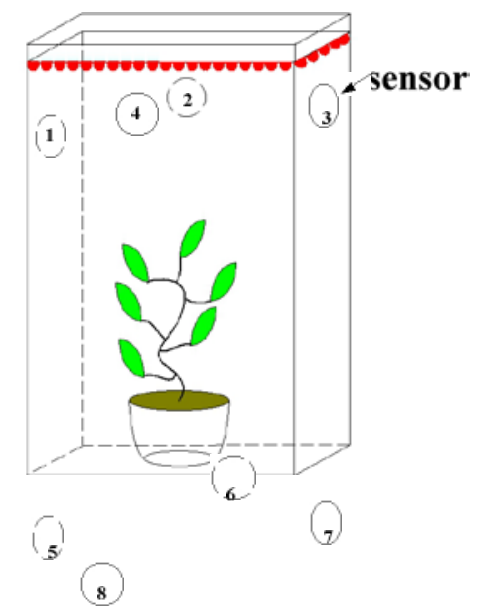

Fig. 2 The structure of plant growth cabinet

\section{The BP neural network}

Multi-layer BP network has an input node, an output node and one or more layers of hidden nodes [3]. The learning process of BP neural network can be divided into two stages: the first stage is the forward propagation process, the input signal via the input layer and hidden layer that it is processing, through the output layer after calculating the output value is obtained. The second stage is the error back-propagation process, if in the output layer did not get the expected output value, per layer recursive calculated the difference between the actual output and the expected output, and adjust the weight coefficient according to this error. Three layer BP neural network structure is shown in Fig. 3.

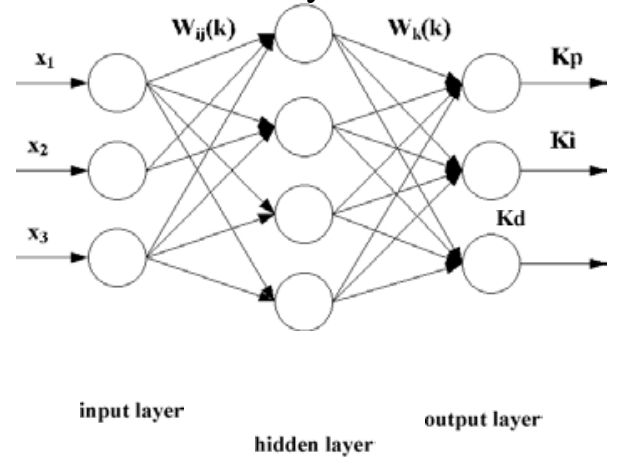

Fig. 3 Three layer BP neural network structure

\section{PID controller of BP neural network}

PID is a linear controller. It gives a deviation of control system according to the given input and the actual output value:

$$
e(t)=c(t)-r(t)
$$


It is called a PID controller that the deviation of the ratio of P, integral I, differential D constituted by a linear combination of the control amount of the controlled object. The computer control system uses digital PID controller. Classic incremental digital PID control equation is:

$$
u(t)=u(k-1)+K_{p}[e(k)-e(k-1)]+T_{i} e(k)+T_{d}[e(k)-2 e(k-1)+e(k-2)]
$$

In the formula: $K_{p} 、 T_{i} 、 T_{d}$ are coefficient of proportional, integral, differential.

The key issue about the design of PID controller is how to choose the proportional, integral, differential coefficient, and these parameters tuning difficult that the application of PID controller is limited. It has the weakness of classical control theory, so only for the constant system of general linear has better control effect.

In order to overcome the weaknesses of conventional PID control, the control community has made a number of improvements on the PID control scheme. PID control of BP neural network is an improvement and optimization of conventional PID control.

BP neural network has ability to approximate any nonlinear function ${ }^{[4]}$, and the structure and learning algorithm is simple and clear, so in the network structure of PID control of BP neural network is often used to build PID controller. BP neural network through their own learning, you can find P, I, D parameters under certain optimal control law. PID controller of BP neural network consists of two parts: the classic PID controller and neural network. Which neural network based on the operational of the system, the output layer neuron output corresponds to the PID controller 3 adjustable parameters $K_{p} 、 T_{i} 、 T_{d}$. Through the neural network self-learning, adjusting the coefficients, it corresponds to the optimal control of PID controller parameters in the stable. A system configuration diagram of PID controller based on neural network is shown in Fig.4.

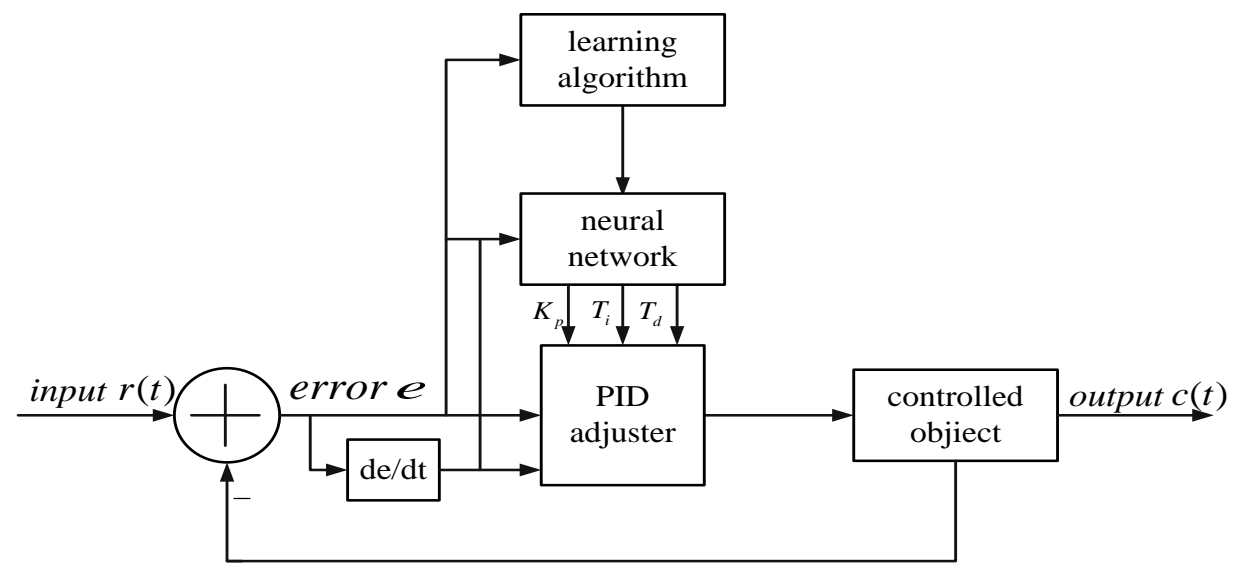

Fig. 4 PID controller based on BP neural network

\section{Simulation research}

This paper takes the greenhouse production of common leafy vegetables -- lettuce for example ${ }^{[5]}$. Lettuce is suitable for growing in cool place, studies showing that the most suitable for the growth temperature is $15-25^{\circ} \mathrm{C}$. The most suitable temperature of the cabbage is between $10-16^{\circ} \mathrm{C}$. When the temperature is higher than $25^{\circ} \mathrm{C}$, the ball leaves may be necrotic or rot due to the high temperatures inside, further making the poor growth. Observe the temperature changes in a day, every 30 minutes a time temperature measurement.

Using MATLAB to simulate the environment for the growth of lettuce, which we give the initial greenhouse control various environmental parameters are not applied. The greenhouse air temperature is $16{ }^{\circ} \mathrm{C}$. The MATLAB simulation map was shown in Fig. 5. 


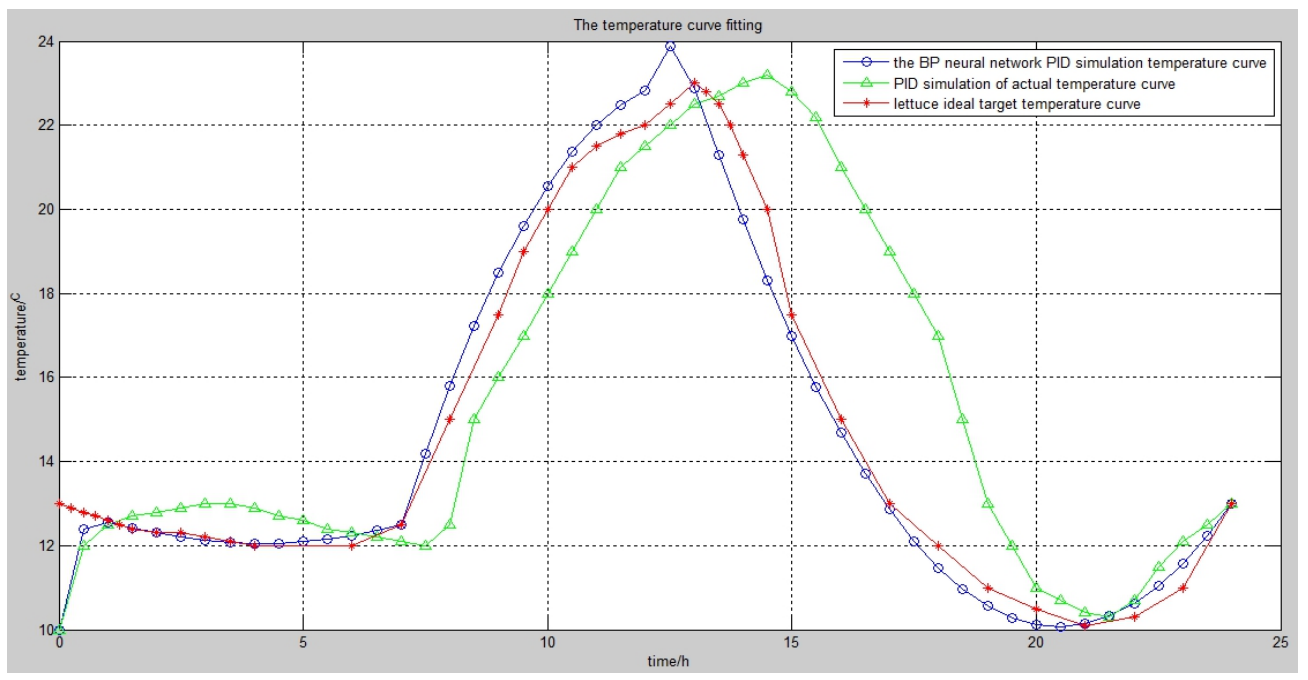

Fig. 5 MATLAB simulation map

From the Fig. 5 we can see that the greenhouse`s temperature controlled by PID control of BP neural network can keep pace with the target temperature curve after rise from $10^{\circ} \mathrm{C}$ to $12.5^{\circ} \mathrm{C}$.

The figure shows the rise time is 0.85 hours approximately, after tracking the target curve to achieve a certain degree. Because the PID control have a certain delay feature, the control effect is relatively worse. PID control of BP neural network was substantially overcome its latency, shorten the adjustment time and reduced overshoot compared with the traditional PID control, and better controlled temperature changing with the target temperature.

\section{Conclusions}

In this paper, combined BP neural network with conventional PID, PID temperature control of BP neural network applied in intelligent plant growth cabinet. It also compared with the conventional PID control by simulation. From image comparison analysis, PID control of BP neural network has fast response speed, not obvious delayed, small overshoot and good stability. Better control temperature changes with the target curve, so that the temperature basically following the target temperature.

\section{References}

[1] Gaoli Chen, Chenfang Ji. A Method for the Measurement of Temperature Based on Multisensor Data Fusion. 2016 4th International Conference on Advanced Materials and Information Technology Processing, 2016 (9): 457-460.

[2] S. I. Some artificial neural network application to greenhouse environmental control. Computers and Electronics in Agriculture, 1997 (2):167-186.

[3] T. L, Satty. Decision making-the analytic hierarchy and network process. Journal of Systems Science and Systems Engineering, 2004.01: 23-27.

[4] Jia He, Zhenghua Da. Implementation of BP neural network PID controller simulation. Journal of Gansu Lianhe University, 2005, 4(2): 31-33. (In Chinese)

[5] D. Stromberg, M. Andersson, F. Lantz. On plant form-based sensor management. Proceedings of the fifth International Conference on Information Fusion, 2002 (1): 600-607. 